\title{
A Novel Reservation-Based MAC Scheme for Distributed Cognitive Radio Networks
}

\author{
Miguel Luís, Rodolfo Oliveira, Senior Member, IEEE, Rui Dinis, Senior Member, IEEE, \\ and Luis Bernardo, Member, IEEE
}

\begin{abstract}
This paper presents a novel medium access control medium access control (MAC) for distributed single-channel cognitive radio networks (CRNs) denominated cognitive radio reservation MAC (C2RMAC). C2RMAC is intended to be adopted by the nonlicensed users and introduces a double stage scheme to schedule each node's transmission. In this way, C2RMAC increases the use of the spectrum left free by the licensed users, when compared with other protocols. An important contribution of this paper is the assumption of a heterogeneous spectrum sensing condition, i.e., the assumption that different nonlicensed users may sense different levels of channel occupancy. We derive an analytical model to compute the performance of the proposed protocol by adopting innovative concepts to tackle the heterogeneous sensing condition. Several simulation results, including the aggregated throughput and the packet service time, evaluate the performance of C2RMAC and successfully validate the proposed model. Finally, C2RMAC is compared with other state-of-the-art cognitive radio MAC protocols, showing the effectiveness of the proposed solution.
\end{abstract}

Index Terms-Cognitive radio (CR), medium access control, modeling and performance analysis.

\section{INTRODUCTION}

C OGNITIVE radio networks (CRNs) are an effective solution to alleviate the increasing demand for radio spectrum [1]. In these networks, nonlicensed users, i.e., usually denominated secondary users (SUs), must be aware of the activity of the licensed users, denominated primary users (PUs), to dynamically access the spectrum without causing harmful interference to PUs. In decentralized cognitive radio networks (DCRNs), the SUs are not managed by a central coordinator, meaning that SUs must adopt distributed policies able to manage the network in an efficient way.

This paper considers a single-radio CRN, where SUs are only equipped with a single transceiver, and a single-channel scenario is assumed. Multiple SUs in communication range of each other

Manuscript received November 9, 2015; revised March 4, 2016 and June 24, 2016; accepted August 3, 2016. Date of publication September 2, 2016; date of current version May 12, 2017. This work was supported in part by the Portuguese Science and Technology Foundation (FCT/MEC) under Project UID-EEA-50008-2013. The review of this paper was coordinated by Prof. G. Mao.

M. Luis is with the Instituto de Telecomunicações, Lisboa 1049-001, Portugal (e-mail: nmal@campus.fct.unl.pt).

R. Oliveira, R. Dinis, and L. Bernardo are with the Instituto de Telecomunicações, Lisboa 1049-001, Portugal, and also with the Departamento de Engenharia Electrotécnica, Faculdade de Ciências e Tecnologia, Universidade Nova de Lisboa, Caparica 2829-516, Portugal (e-mail: rado@fct.unl.pt; rdinis@fct.unl.pt; lflb@fct.unl.pt).

Color versions of one or more of the figures in this paper are available online at http://ieeexplore.ieee.org.

Digital Object Identifier 10.1109/TVT.2016.2605718 wish to transmit in an opportunistic way when the PUs do not use the channel. The SU receiver (SU Rx) may be for example an access point, but since the proposed protocol operates in a distributed way, each SU may be a hypothetical receiver. In this way, the proposed scheme may be adopted in DCRNs using wide-band channels, which is currently a realistic scenario motivated by the opportunistic General Authorised Access [2] recently proposed by the Federal Communications Commission (FCC)

The SUs synchronously and periodically sense the channel to determine the level of PU's activity. The spectrum is sensed using an energy-based sensing (EBS) scheme, but more sophisticated spectrum sensing techniques may be adopted, such as the ones described in [3]. The operation of the SUs is organized in frames. Each frame is organized in two periods. The spectrum sensing is performed in the first period in order to evaluate if the channel is being used by PUs. In the second period, a SU employs the proposed cognitive radio reservation medium access control (MAC) (C2RMAC), when no PU is detected during the sensing period. Otherwise a SU suspends any activity during the second period and waits for a future decision in the sensing period of the next frame. C2RMAC relies on two stages. The first stage lasts a single frame and is used to decrease the probability of collision between SUs, by reducing the number of competing SUs. The second stage starts with a reservation phase where SUs may schedule their transmissions, and finishes with the transmission phase, where the SUs effectively access the channel.

To the best of our knowledge, this is the first cognitive radio (CR) MAC protocol for DCRNs based on a reservation scheme, which can be particularly advantageous when high dissimilarity of spectrum sensing decisions achieved by the different SUs is observed in a given frame. When this occurs the number of competing SUs is a time-varying parameter and the reservation scheme can efficiently accommodate the competing SUs by dynamically varying the number of reserved frames according to the number of SUs requesting for transmission. By this way, we avoid the underperformance caused by the use of traditional random contention schemes, namely, when the level of contention does not take the number of competing nodes into account.

The main contributions of this paper are listed as follows:

1) We assume individual channel sensing heterogeneity, i.e., different SUs may achieve different sensing outcomes, making the number of SUs competing for the medium a time-varying parameter. As far as we know, this is the first work to handle such scenario, which increases the 
complexity of the MAC protocol modeling task. Sensing heterogeneity may represent both noncooperative sensing or even cooperative sensing, where the nodes have different views of the channel availability.

2) We derive an analytical model for the service time achieved by the protocol considering heterogeneous channel sensing. The model relies on two independent Discrete Time Markov Chains (DTMCs), which model the behavior of a transmitting SU, and the operating mode of the SU responsible for the synchronization of the protocol, respectively. The individual throughput achieved by a SU is characterized and validated.

3) We propose a formal analysis for the synchronization of the two independent DTMCs considered in 2). It is well known that the steady-state probabilities of a synchronization state in different DTMCs are not equal when the DTMCs have different probability spaces [4]. By characterizing the discrete time distribution of specific states in each DTMC, we derive the probability of synchronization through the comparison of the time distributions of the synchronizing states of both DTMCs.

4) Finally, we provide a comparison of the performance achieved by C2RMAC for different network sizes and levels of primary activity.

In the next section, we review a few works related to ours. Section III introduces C2RMAC protocol. A formal analysis of the protocol considering heterogeneous spectrum sensing is derived in Section IV. Performance results are discussed in Section V and concluding remarks are given in Section VI.

\section{RELATED WORK}

The first MAC schemes proposed for DCRNs considered multichannel networks, where the number of channels available for each SU varies in time and in space. The access of the SUs to the different channels may be coordinated by a common control channel (CCC) [5]-[9], which requires the use of two radios (one for signaling and another for data transmission). The adoption of a single radio is also allowed in the so-called Split Phase protocols [10], in which a first time interval (period) of the frame is used for signaling and control, and a second period is used for data transmission when the channel is vacant. Other protocols, such as the standard IEEE 802.22 [11] and the protocol described in [12], avoid the use of a control channel by handling signaling and data transmission in the same channel (in-band signaling). Shrestha et al. [13] proposed a hybrid CSMA/CATDMA access policy for single-hop wireless networks, which is able to cope with the network congestion due to increased traffic load. However, all these protocols adopt a centralized architecture, where a central node (e.g., a base station) coordinates the channel access [11], [13] or manages the spectrum sensing [12].

Much less work has been done in decentralized split phase protocols. Random access protocols were proposed to implement SU's distributed channel access [14]-[16]. In [14], each SU starts to sense the channel before trying to transmit data. If the channel is sensed idle, a random backoff is employed and the channel is granted to the first SU finishing the backoff period.
Lien et al. [15] proposes the use of a carrier sense multiple access (CSMA) scheme. CSMA and Slotted Aloha-like medium access policies were also proposed in [16]. CR-ALOHA and CR-CSMA were proposed to deal with the packet scheduling of the secondary network. However, CR-ALOHA and CR-CSMA exhibit low performance, namely, due to the protocol's design. This fact is confirmed in [17], which proposes a CSMA scheme with collision avoidance (CR-CSMA/CA) similar to the contention regulation applied in IEEE 802.11. While the previous works in [14]-[17] can be classified as decentralized Split-Phase protocols, they are based in random contention, which may lead to low performance, because these protocols trade off between the minimization of the probability of collision and the probability of finding idle medium access slots [18].

Reservation-based schemes have been proposed in several wireless systems to reduce the number of idle slots needed to schedule a transmission [19]-[21]. Zhao et al. in [20] and [21], adopt ready-to-send and clear-to-send packets to reserve the transmission. However, the asynchronous nature of these protocols is not indicated for the proposed DCRN scenario. Hsu and $\mathrm{Su}$ [22] evaluate the throughput region of a centralized reservation-based access scheme. Regarding the CRNs, most of the reservation-based MAC schemes addressed in the literature [10], [23]-[27] are focused on multichannel scenarios or demand for an extra channel (CCC) for signaling exchange. For example, in [25] and [26], two reservation-based MAC schemes are presented where each SU is equipped with two transceivers: while in [26], the SUs perform the reservation in a dedicated CCC, in [25], the SU's frame is divided into many slots as the number of data channels.

In this paper, we focus on a decentralized Split-Phase protocol where, contrarily to [5]-[13], neither a CCC nor a centralized architecture is required. Instead of adopting a random contention medium access strategy as in [14]-[18], the proposed protocol uses a reservation-based access scheme. While reservation-based access protocols are known to increase the throughput through the reduction of the number of idle slots needed to schedule a transmission, they only have been proposed to CRNs where a CCC or a centralized architecture is adopted [10], [23]-[26]. This is mainly justified by the easiness of coordination of the channel reservation task in a centralized architecture, when compared to a decentralized one. Proposed for a decentralized architecture, CR2MAC benefits from the reservation-based access supported by a double stage scheme to schedule each nonlicensed user's transmission. Although only a small number of Split-phase protocols have been proposed for decentralized CRNs [14]-[17], the results achieved with C2RMAC reveal that the adoption of the double stage reservation scheme comparatively increases the network's throughput. The improvement is due to the fact that the first stage reduces the probability of collision between nonlicensed users, while the second stage reduces the number of idle frames usually left unused by the secondary network. A preliminary characterization of some concepts adopted in C2RMAC was presented in [28], which evaluates the impact of the channel sensing heterogeneity in the throughput of a distributed reservation-based MAC. While the characterization in [28] is only based on simulation results, in this paper, we are focused on the theoretical modeling 


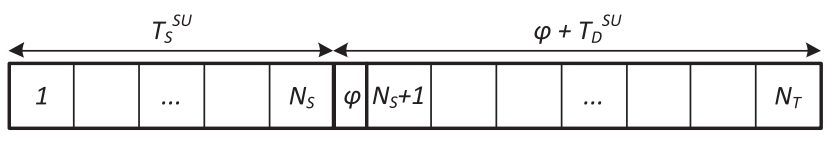

Fig. 1. SU's frame structure.

of the throughput and service time under heterogeneous channel sensing conditions.

\section{C2RMAC PROTOCOL}

\section{A. Protocol's Time Framing}

In this paper, we consider a single-hop network where $n$ SUs may transmit data in an opportunistic way, when the channel is not being used by PUs. The proposed MAC scheme works in a distributed way, without being coordinated by a central node. C2RMAC can be adopted in a scenario where each node transmits to a random destination, by specifying the destination address in the header of the data frame. However, because in a single-hop network, the destination nodes are within the communication range of the transmitters, in the theoretical characterization we consider that the SUs always transmit to the same $\mathrm{SU}$, which is referred to as a SU Rx. Finally, we admitted that each $\mathrm{SU}$ is equipped with a single radio transceiver.

SUs are unable to distinguish SUs and PUs transmissions. Therefore, each SU divides its operation cycle (frame structure) into spectrum sensing and spectrum access periods, with durations $T_{S}^{\mathrm{SU}}$ and $T_{D}^{\mathrm{SU}}$ respectively, as illustrated in Fig. 1. A frame lasts for $T_{F}^{\mathrm{SU}}=T_{S}^{\mathrm{SU}}+\varphi+T_{D}^{\mathrm{SU}}$ and is divided into $N_{T}$ slots, where each slot duration is given by the channel sampling period [29]. $\varphi$ is a time interval needed to synchronize the SUs. The first $N_{S}$ slots are allocated for spectrum sensing (channel sampling) and the remaining ones $\left(N_{S}+1\right.$ to $\left.N_{T}\right)$ are used to synchronization and channel access (transmission). Initially, an SU assumes the synchronization task if it does not receive synchronization information during a predefined time interval. Such an SU is called synchronizer and is responsible for the transmission of a short synchronization packet during the $\varphi$ interval containing the duration of the spectrum sensing and spectrum access periods. The synchronization (SYNC) packet is transmitted by the synchronizer whenever the channel is sensed idle during the spectrum sensing period. By receiving the SYNC packet, the neighbors (denoted as followers) know the duration of the spectrum sensing and spectrum access periods and may correct clock drifts. If the synchronizer SU does not transmit the SYNC packet within a given period of time denoted as SYNC_TIMEOUT (e.g., because that SU has finished its activity), any follower may then assume that role. To become a synchronizer, a follower is allowed to randomly transmit the SYNC packet after the SYNC_TIMEOUT has been elapsed. Randomness is used to avoid multiple nodes acting simultaneously as synchronizers. This is similar to the synchronization schemes already proposed for distributed MAC schemes of wireless sensor networks, where any node can act as a synchronizer [30]. For the sake of simplicity of the analysis, in what follows, we consider that the SU Rx is always a synchronizer, being responsible for the synchronization of the SUs. In the protocol, however, we highlight that the syncronizer SU may be any one of the SUs, and it may change over time.

\section{B. Spectrum Sensing}

In this paper, we consider that SUs and the SU Rx sense the channel during the period $T_{S}^{\mathrm{SU}}$ using an EBS technique [31]. To distinguish between occupied and vacant spectrum bands, SUs and the SU Rx sample, the channel during the sensing period $T_{S}^{\mathrm{SU}}$, and the received energy $Y$ is compared with a predetermined threshold $\theta$. Based on the result, the spectrum may be declared idle $(Y<\theta)$ or busy $(Y \geq \theta)$. The performance of the energy detector is determined by the probability of detection $P_{D} \approx \mathcal{Q}\left(\frac{\theta-\left(N_{S}+\lambda\right)}{\sqrt{2\left(N_{S}+2 \lambda\right)}}\right)$, i.e., the probability that $Y \geq \theta$ when the spectrum is being used by PUs, and the probability of false alarm $P_{F A} \approx \mathcal{Q}\left(\frac{\theta-N_{S}}{\sqrt{2 N_{S}}}\right)$, which is the probability that $Y \geq \theta$ when the channel is actually idle, where $\mathcal{Q}($.$) is the complementary distri-$ bution function of the standard normal distribution and $\lambda$ denotes the signal-to-noise-ratio (SNR) [32].

SUs may declare an idle or busy spectrum access period with duration $T_{D}^{\mathrm{SU}}$, if the spectrum sensing applied during the $N_{S}$ slots indicates absence or presence of a PU, respectively. SUs start to decide their medium access from the moment when the current frame is declared idle or busy. After collecting $N_{S}$ samples, the adopted sensing scheme decides the spectrum occupancy status. Considering that $P_{\mathrm{ON}}^{\mathrm{PU}}$ represents the probability of having a PU currently transmitting in the channel, and $P_{\mathrm{OFF}}^{\mathrm{PU}}$ the opposite case, the probability of a SU deciding that the channel is vacant is given by

$$
P_{I}=P_{\mathrm{OFF}}^{\mathrm{PU}}\left(1-P_{\mathrm{FA}}\right)+P_{\mathrm{ON}}^{\mathrm{PU}}\left(1-P_{D}\right)
$$

and different notations $P_{I, i}$ and $P_{I, R x}$ are introduced for the probability $P_{I}$ of the $i$ th $\mathrm{SU}$ and the SU Rx, respectively. $P_{I}$ accounts with the sensing accuracy of each SU, by including the EBS' individual probabilities of misdetection $\left(1-P_{D}\right)$ and correct PU absence rejection $\left(1-P_{F A}\right)$. From hereafter we denote as idle frames, the frames where the spectrum sensing scheme does not detect any PUs' activity with probability $P_{I}$, and as busy frames the opposite case that occurs with probability $1-P_{I}$. Consequently, the interference caused to PUs only depends on the accuracy of the adopted spectrum sensing technique.

\section{Details of C2RMAC Protocol}

Fig. 2 introduces C2RMAC protocol, in which a transmission cycle comprises two stages of contention. The SU Rx indicates the current stage of contention by transmitting control packets during the synchronization time interval $\varphi$. The first stage of contention aims to decrease the number of collisions between SUs by reducing the number of competing SUs. This stage lasts a single idle frame (firstframe in the example depicted in Fig. 2), being its spectrum access period $T_{D}^{\mathrm{SU}}$ divided in $c w_{1}$ mini-slots. ${ }^{1}$ In the beginning of the first stage, the SUs

\footnotetext{
${ }^{1}$ Note that we use the term mini-slots for MAC purposes, while the term slot adopted in Section III-B is only used for channel sensing purposes.
} 

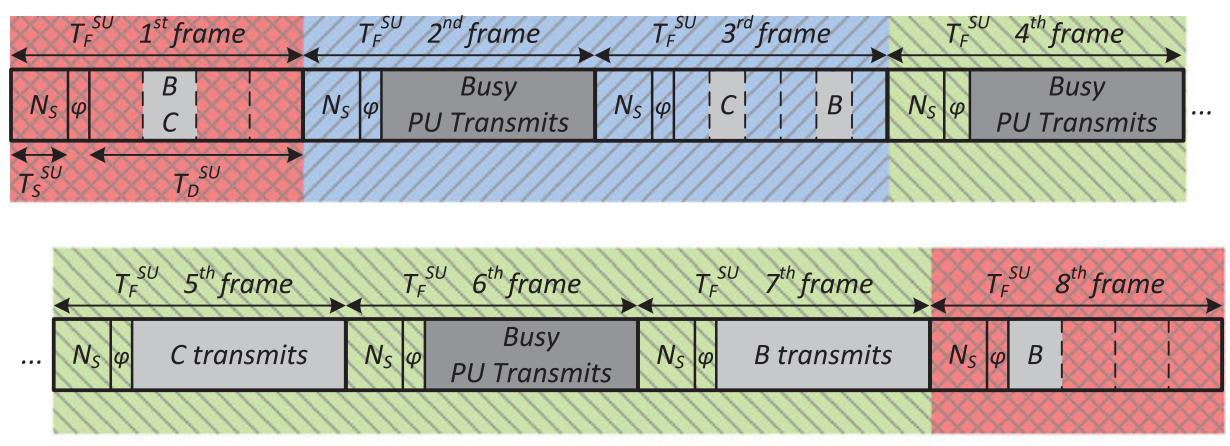

$1^{\text {st }}$ Stage of contention Reservation phase of the $2^{\text {nd }}$ stage Transmission phase of the $2^{\text {nd }}$ stage

Fig. 2. C2RMAC transmission cycle $\left(c w_{1}=4\right.$ and $\left.c w_{2}=6\right)$.

randomly select a minislot with probability $\tau_{1}=1 / c w_{1}$, which serves to announce its intention to access the medium. However, a SU will only transmit its minipacket if the previous minislots were found idle, i.e., if a SU senses a SU's transmission in a minislot before the randomly selected one, it will postpone the transmission attempt to the next transmission cycle. According to this rule, only the SUs that have transmitted a minipacket in the first stage will be able to compete in the second stage. In Fig. 2, SUs B and C transmit a minipacket in the second minislot of the firstframe, while SU A will not transmit its minipacket scheduled for the fourth minislot because SUs B and $\mathrm{C}$ have already transmitted. This means that only SUs B and C will compete in the second contention stage, since they were the first nodes accessing a minislot in the frame. ${ }^{2}$

Nodes selected in the first stage will be able to compete in the second stage of contention. The spectrum access period $T_{D}^{\mathrm{SU}}$ of the first idle frame in the second stage is divided into $c w_{2}$ minislots $\left(c w_{2}=6\right.$ in this case), which are used to reserve at most $c w_{2}$ future idle frames for transmission. This is called the reservation phase of the second stage. Admitting that $n_{\mathrm{St} 2}$ SUs compete for medium access in the second stage, they reserve an idle frame by transmitting a minipacket in one of the frame's minislots $\left\{1,2, \ldots, c w_{2}\right\}$. Each SU chooses a minislot in the second stage with probability $\tau_{2}=1 / c w_{2}$. In the example illustrated in Fig. 2, the second stage of contention begins in the secondframe; however, the reservation phase only occurs in the next idle frame, which is the thirdframe. The SU C transmits a minipacket in the second minislot and the SU B transmits its minipacket in the fifth minislot. The number of idle frames reserved for future transmission is equal to the number of busy minislots, and the assignment of the idle frames follows the sequence of accesses observed in the $c w_{2}$ minislots. Because the protocol is decentralized, each SU assigns itself an idle frame, i.e., if a SU transmits a minipacket in the $k$ th busy minislot, $1 \leq k \leq c w_{2}$, it reserves the subsequent $k$ th idle frame for its own transmission. ${ }^{3}$ Implicitly, it is assumed that if multiple SUs

\footnotetext{
${ }^{2}$ For the sake of simplicity, PU's frames were illustrated having the same size as SU's frame.

${ }^{3}$ To this purpose, it is assumed that the SUs are able to detect a transmission of one or more mini-packets in a minislot before the one where they access, which is nowadays a reality in every MAC protocol following a carrier sensing approach.
}

access in the same minislot, they will collide in the reserved frame. This fact is taken into account in the proposed analysis.

After elapsing the $c w_{2}$ minislots of the reservation phase, the SUs use the reserved frames to transmit, and this period is denominated transmission phase of the second stage. In the example illustrated in Fig. 2, the first idle frame occurring after the thirdframe is reserved for the transmission of node $\mathrm{C}$, which occurs in the fifth frame illustrated in Fig. 2. The same follows for the reservation done by node B, which transmits in the seventh frame. The transmission cycle lasts for the first seven frames and a new transmission cycle begins in the eighth frame. Neglecting the number of busy frames occupied by PU's transmissions (second, fourth, and sixth frame in the example), the second stage lasts for the number of idle frames equal to the number of busy minislots observed in the reservation phase ( $f$ ifth and seventhframes), plus the idle frame where the $c w_{2}$ minislots were defined (see thirdframe).

\section{Analytical Performance Model}

This section analyses the performance of the C2RMAC protocol considering an heterogeneous spectrum sensing scenario, i.e., for the same spectrum sensing period, all SUs (including the SU Rx) may obtain different spectrum sensing outcomes. This means that in the worst case spectrum decisions achieved by each SU during the same spectrum period may be independent of each other. Therefore, at each moment the operation of each SU and SU Rx may be in different MAC states, requiring that the MAC states of the SU Rx and the MAC states of each SU must be modeled in an independent manner. For that, we have decided to resort to DTMCs: one DTMC to model the SU Rx and another DTMC to model the individual behavior of each SU.

Before going into the details of each DTMC, let us overview the protocol's operation when a SU and the SU Rx obtain different spectrum sensing outcomes for the same spectrum sensing period. When this occurs, the following scenarios must be considered.

1) The channel is busy for the SU Rx and idle for an SU.

a) If the protocol is in the reservation phase, the $\mathrm{SU}$ $\mathrm{Rx}$ will not transmit the packet regarding the first 


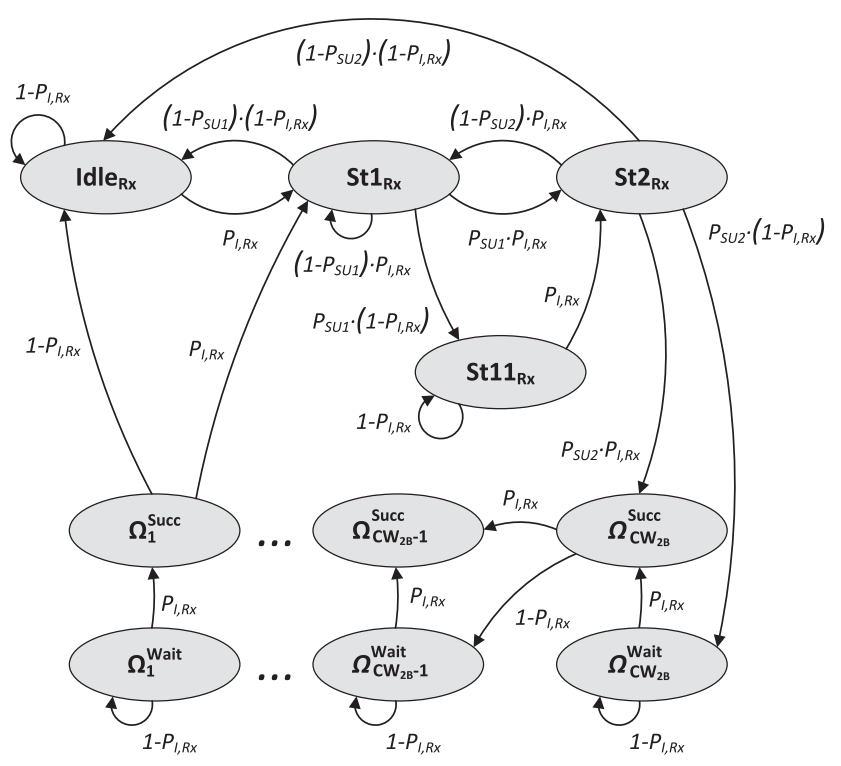

Fig. 3. DTMC illustrating the SU Rx's operation mode.

and the second stage, and consequently, the SU will not compete for the channel.

b) If the protocol is in the transmission phase, the SU will transmit, and the transmission will not be considered for throughput because the channel is busy for the SU Rx.

2) The channel is busy for a SU and idle for the SU Rx.

1) In this case, whether the protocol is in the reservation's phase or in the transmission's phase, the SU will lose the opportunity to compete or to transmit, while other SUs sensing the channel as being idle will advance with the protocol's operation.

\section{A. SU Rx's DTMC}

The DTMC illustrated in Fig. 3 models the SU Rx operation cycle. In the beginning of the cycle, the SU Rx is initially at state Idle $_{\mathbf{R x}}$. After sensing an idle frame, with probability $P_{I, \mathrm{Rx}}$, the SU Rx proceeds to the first stage of contention $\left(\mathbf{S t} \mathbf{1}_{\mathbf{R x}}\right)$, where it will remain while the frames are declared idle and no SUs compete for the first stage (with probability $1-P_{\mathrm{SU} 1}$ ).

While in $\mathbf{S t} \mathbf{1}_{\mathbf{R x}}$, if a busy frame is detected without having SUs competed in the first stage, the SU Rx will return to the initial idle state (Idle $\mathbf{R}_{\mathbf{R x}}$ ). In the case of having SUs competing in the first stage, which occurs with probability $P_{\mathrm{SU} 1}$, the SU Rx will proceed to the second stage $\left(\mathbf{S t}_{\mathbf{R x}}\right)$ in the next idle frame, or proceed to state $\mathbf{S t 1 1} \mathbf{R x}_{\mathbf{R x}}$ as busy frames are detected. Because it is assumed that SUs and the SU Rx may experience different spectrum sensing results, it can happen that during the stage St $2_{\mathbf{R x}}$ all the SUs selected to compete in the second stage may declare a busy frame, and no one will compete. The probability of not having SUs competing in the second stage is given by $1-P_{\mathrm{SU} 2}$. This situation makes the SU Rx restart the operation cycle by going from state $\mathbf{S t} \mathbf{2}_{\mathbf{R x}}$ back to $\mathbf{S t} \mathbf{1}_{\mathbf{R x}}$ or $\mathbf{I d l e} \mathbf{R}_{\mathbf{R x}}$ in the next frame, depending on if the frame is declared idle or busy, respectively.
In the case of having SUs competing in the second stage, which occurs with probability $P_{\mathrm{SU} 2}$, the $\mathrm{SU} \mathrm{Rx}$ starts the transmission phase, which will be used by the SUs to communicate with the SU Rx. The number of busy minislots in the reservation phase is denoted by the random variable $C W_{2 B}$. The transmission phase starts when a transition from $\mathbf{S t} \mathbf{2}_{\mathbf{R x}}$ to $\Omega_{\mathrm{CW}{ }_{2 \mathrm{~B}}}^{\mathrm{Wait}}$ or $\Omega_{\mathrm{CW}_{2 \mathrm{~B}}}^{\mathrm{Succ}}$ is observed. During the transmission phase, the SU Rx has to wait for $C W_{2 B}$ idle frames before completing the operation cycle: after observing $C W_{2 B}$ idle frames, the $\mathrm{SU}$ Rx reaches the state $\Omega_{1}{ }^{\text {Succ }}$, restarting the cycle from Idle $\mathbf{R}_{\mathbf{R x}}$ or $\mathbf{S t} \mathbf{1}_{\mathbf{R x}}$, depending on the spectrum status.

The formal treatment of heterogeneous spectrum opportunities raises several issues that do not occur when all SUs and the SU Rx share the same view of the channel's occupancy. The biggest one is to guarantee that each $\mathrm{SU}$ is doing the right action in the corresponding frame, e.g., to assure that a SU does not compete in the second stage in frames representing the first stage of contention (state $\mathbf{S t} \mathbf{1}_{\mathbf{R x}}$ ). For that, we assume that the SU Rx transmits two different packets, during the synchronization period $\varphi$, one in each stage frame represented by the states $\mathbf{S t} \mathbf{1}_{\mathbf{R x}}$ and $\mathbf{S t} \mathbf{2}_{\mathbf{R x}}$. This way, all the SUs have the possibility to get aligned with the SU Rx, when they receive the packet indicating the beginning of the first stage of contention (state $\mathbf{S t}_{\mathbf{R x}}$ ) or the beginning of the second stage of contention (state $\mathbf{S t} \mathbf{2}_{\mathbf{R x}}$ ).

Let $\left\{R_{k}\right\}_{k \geq 0}$ be a discrete-time stochastic process representing the generic $\zeta \mathrm{MAC}$ state of the $\mathrm{SU} \mathrm{Rx}$ at frame $k$, with $\zeta \in$ $\mathcal{G}=\left\{\mathbf{I d l e}_{\mathrm{Rx}}, \mathbf{S t}_{\mathrm{Rx}}, \mathbf{S t}_{1} 1_{\mathrm{Rx}}, \mathbf{S t} 2_{\mathrm{Rx}}, \Omega_{\mathrm{CW}_{2 \mathrm{~B}}}^{\mathrm{Wait}}, \Omega_{\mathrm{CW}_{2 \mathrm{~B}}}^{\mathrm{Succ}_{2}}, \ldots\right.$, $\left.\Omega_{1}^{\text {Wait }}, \Omega_{1}^{\text {Succ }}\right\}$. The matrix $\mathcal{P}^{\mathrm{Rx}}=\left\{\mathcal{P}_{\zeta_{A}, \zeta_{B}}^{\mathrm{Rx}}\right\}_{\zeta_{A}, \zeta_{B} \in \mathcal{G}}$ denotes the $|\mathcal{G}| \times|\mathcal{G}|$ transition matrix of the stochastic process $R$, where $|$.$| denotes the cardinality of a set, and with \mathcal{P}_{\zeta_{\mathcal{A}}, \zeta_{\mathcal{B}}}^{\mathrm{Rx}}=$ $\operatorname{Pr}\left\{R_{k+1}=\zeta_{B} \mid R_{k}=\zeta_{A}\right\}$ representing the probability regarding the single-step transition from the generic state $\zeta_{A}$ to $\zeta_{B}$. Since $\mathcal{P}_{\zeta_{\mathcal{A}}, \zeta_{B}}^{\mathrm{Rx}}$ is independent of $k$, and the Markov property can be applied, then $R$ is said to be a homogeneous DTMC. Moreover, if a stationary distribution $\pi$ with respect to the DTMC $R$ exists, for $\zeta, \chi \in \mathcal{G}$, the following conditions hold:

$$
\begin{aligned}
& 0 \leq \pi_{\zeta} \leq 1 \\
& \sum \pi_{\chi} \cdot \mathcal{P}_{\chi, \zeta}^{\mathrm{Rx}}=\pi_{\zeta} \\
& \sum \pi_{\zeta}=1
\end{aligned}
$$

and the steady-state distribution of state $\zeta$ is given by $\pi_{\zeta}$. Considering that the DTMC $R$ is aperiodic and positive recurrent, the expected value of the return time to a given state $\zeta \in \mathcal{G}$, also know as regenerative time, is given by $\left[\pi_{\zeta}\right]^{-1}$, as derived in Appendix A. Therefore, and following the classical definition of relative frequency of occurrence of an event, we can approximate the probability of having a SU $i$ competing in the first stage of contention as follows:

$$
P_{\mathrm{SU} 1, i}=\frac{\left[\pi_{\mathrm{St} 1_{\mathrm{Rx}}}\right]^{-1}}{\left[\pi_{\mathrm{St} 1_{\mathrm{SU}, \mathrm{i}}}\right]^{-1}}=\frac{\pi_{\mathrm{St} 1_{\mathrm{SU}, \mathrm{i}}}}{\pi_{\mathrm{St} 1_{\mathrm{Rx}}}}
$$

where $\left[\pi_{\mathrm{St}_{1} \text { SU, }_{\mathrm{i}}}\right]^{-1}$ refers to the regeneration time of the SU $i$ with respect to the first stage of contention, which is defined in Section IV-C. Similarly, $P_{\mathrm{SU} 2, i}$ refers to the probability of 